\section{Model-based Identification of Some Conditions Leading to Glycolytic Oscillations in E. coli Cells}

\author{
G. Maria," M. Mihalachi, and C. Luminita Gijiu \\ Department of Chemical and Biochemical Engineering, \\ University Politehnica of Bucharest, Romania
}

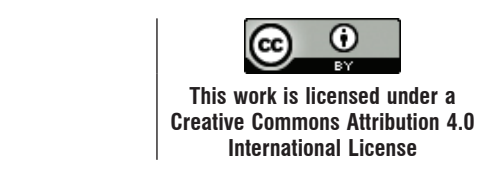

doi: 10.15255/CABEQ.2017.1241

Original scientific paper Received: October 18, 2017 Accepted: December 7, 2018

Autonomous oscillations of glycolytic intermediate concentrations reflect the dynamics of the control and regulation of this major catabolic pathway, and this phenomenon has been reported in a broad range of bacteria. Understanding glycolytic oscillations might therefore prove crucial for the general understanding of the regulation of cell metabolism with immediate practical applications, allowing in silico design of modified cells with desirable 'motifs' of practical applications in the biosynthesis industry, environmental engineering, and medicine. By using a kinetic model from literature, this paper is aiming at in silico (model-based) identification of some conditions leading to the occurrence of stable glycolytic oscillations in the E. coli cells.

Keywords:

reduced dynamic model, glycolysis, Escherichia coli, oscillation occurrence, in silico determination of glycolytic oscillation conditions

\section{Introduction}

Autonomous oscillations of the glycolytic intermediates' concentrations reflect the dynamics of the control and regulation of this major catabolic pathway, and the phenomenon has been reported in a broad range of cell types. ${ }^{1}$ Understanding glycolytic oscillations might therefore prove crucial for our general understanding of the regulation of metabolism and the interplay among different parts of metabolism, as illustrated, for instance, by the hypothesis that glycolytic oscillations play a role in complex pulsatile insulin secretion, ${ }^{2}$ or in the amino-acid synthesis. ${ }^{3}$ In this context, the key question concerns the mechanism(s) of the oscillations but, "despite much work over the last 40 years, it remains unsettled". ${ }^{1}$

Glycolysis is an essential part of the cell metabolism. In fact, glycolysis, together with the phosphotransferase (PTS)-system for glucose transport into the cell, the pentose-phosphate pathway (PPP), and the tricarboxylic acid cycle (TCA), characterize the central carbon metabolism (CCM) ${ }^{4-6}$ The CCM model is the 'core' part of any systematic and structured model-based analysis of the cell metabolism with immediate practical applications, such as target metabolite synthesis optimization, insilico re-programming of the cell metabolism to design new microorganisms for industrial bioprocess optimization, etc. ${ }^{4-12}$

"Corresponding author: G. Maria, email: gmaria99m@hotmail.com
However, to cope with the astronomic complexity of cellular processes, of low observability, involving $\mathrm{O}\left(10^{3}-10^{4}\right)$ number state variables (species conc.), $\mathrm{O}\left(10^{3}\right)$ gene expression transcription factors $\mathrm{TF}$, and $\mathrm{O}\left(10^{4}-10^{5}\right)$ reactions, versatile models of 'building-blocks' like modular constructions, including individual and lumped species and reactions have been developed over decades. ${ }^{5,7}$

In this context, lumped modelling of the bacteria glycolysis is a classical subject but still of high interest, allowing in silico design of modified cells with desirable gene circuits and 'motifs' of practical applications in the biosynthesis industry, environmental engineering, and medicine..$^{8-10}$

Consequently, understanding and simulation of the cell characteristics and environmental conditions leading to an oscillating glycolysis is an old subject, but still of high interest. ${ }^{4}$ To simulate the glycolysis in bacteria, a large number of glycolysis models, of a reduced or extended form, have been proposed over decades (review of $\mathrm{Maria}^{4}$ ). Recently, Maria ${ }^{4}$ proposed a reduced glycolysis model (denoted by $m T R M$ in the E. coli cells, including only 9 species, 7 lumped reactions, and 17 estimable parameters. This model was identified using experimental dynamic data from literature, ${ }^{6,18}$ and has been proved to adequately reproduce the cell glycolysis under steady state, oscillatory, or transient conditions according to the defined glucose input flux, environmental conditions, the total A(MDT)P cell energy resources, and cell phenotype characteristics (related to the activity of enzymes involved in the ATP utilization and recovery system). 
This paper is aiming at using the $m T R M$ model of $\mathrm{Maria}^{4}$ to simulate some conditions leading to glycolytic oscillations in the E. coli cells. Because this model is one of good adequacy, relevant results are expected.

\section{Kinetic model of glycolysis in the E. coli prokaryotic bacteria}

Glycolysis (from an older term with the meaning of glucose degradation) is the metabolic pathway that converts glucose $\left(\mathrm{C}_{6} \mathrm{H}_{12} \mathrm{O}_{6}\right)$ into pyruvate $\left(\mathrm{CH}_{3} \mathrm{COCOO}^{-}+\mathrm{H}^{+}\right)$. The free energy released by the subsequent tricarboxylic acid cycle (TCA) originating from pyruvate is used to form the high-energy molecules ATP (adenosine triphosphate), and NADH (reduced nicotinamide adenine dinucleotide) that support the glycolysis and numerous enzymatic syntheses into the cell. ${ }^{13,14}$

Glycolysis is a determined sequence of ten enzyme-catalyzed reactions (Fig. 1). The intermediates provide entry points to glycolysis. For example, most monosaccharides, such as fructose or galactose, can be converted to one of these intermediates. The intermediates may also be directly useful. For example, the intermediate dihydroxyacetone (DHAP, an intermediate in the reaction of $\mathrm{f} 6 \mathrm{p}$ conversion to $g 3 p$ in Fig. 1) is a source of the glycerol that combines with fatty acids to form fat. Also, NADPH is formed by the pentose-phosphate pathway (PPP), which converts glucose into ribose, which is used in the synthesis of nucleotides and nucleic acids. Pep is also the starting point for the synthesis of essential aminoacids such as tryptophan, cysteine, arginine, serine, etc. ${ }^{15,16}$

Due to the tremendous importance of this metabolic process in simulating the cell CCM, intense efforts have been made both in the experimental study, and in modeling the glycolysis dynamics in Escherichia coli, ${ }^{17-20}$ and other cell types.

On the other hand, to model in detail the dynamics and regulation of glycolysis, which is one of the most complex cellular processes, is a very difficult task, if not impossible. Consequently, a large number of glycolysis reduced or extended kinetic models have been proposed over decades (review of Maria ${ }^{4}$, of a complexity ranging from 18-30 species, included in $48-52$ reactions, with a total of 24-150 parameters. Most of these models are however too complex to be easily used and identified. Besides, with a few exceptions, most of the models cannot satisfactorily reproduce the glycolytic oscillations on a mechanistic basis.

However, starting from an extended reaction pathway and model, and by applying chemical engineering lumping techniques, ${ }^{12,21,22}$ Maria $^{4}$ pro-

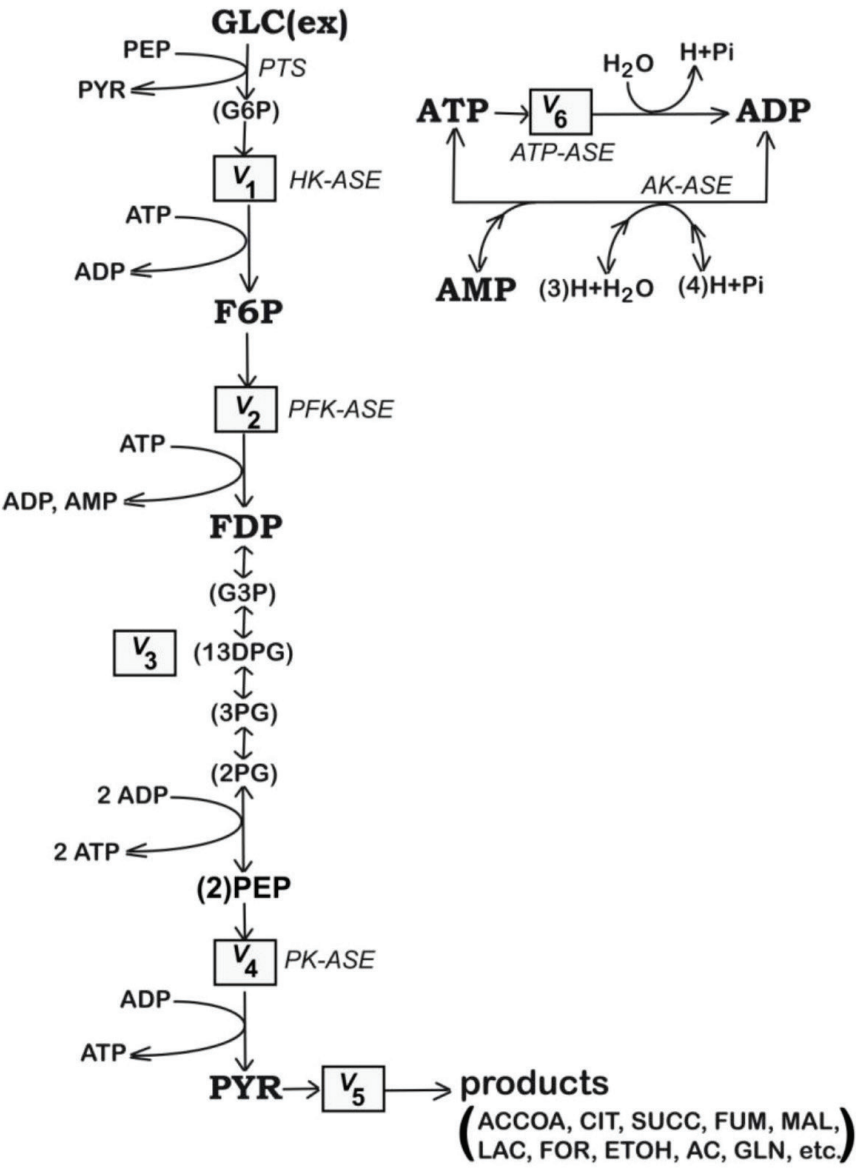

Fig. 1 - Simplified reaction schemes of glycolysis in E. coli to base the reduced kinetic model of Maria ${ }^{4}$ and including adenosin co-metabolites ATP, ADP, AMP synthesis. Species in parenthesis are not explicitly included in the mTRM model. Italic letters denote the enzymes. Squares include notations of enzymatic reactions. Species abbreviations: $g l c(e x)=$ glucose in the cell environment; $g 6 p=$ glucose-6-phosphate; $f 6 p=$ fructose-6-phosphate; HK-ASE - hexokinase; PFK-ASE phosphofructokinase; ATP-ASE = ATP monophosphatase; $A D P=$ adenosin-diphosphate; $A T P=$ adenosin-triphosphate; $A M P=$ adenosin-monophosphate; $A K-A S E=$ adenylate kinase; $P i=$ Phosphoric acid; fdp = fructose-1,6-biphosphate; $g 3 p$, gap $=$ glyceraldehyde-3-phosphate; 13dpg, pgp = 1,3-diphosphoglycerate; $3 \mathrm{pg}=3$-phosphoglycerate; $2 \mathrm{pg}=2$-phosphoglycerate $;$ pep = phosphoenolpyruvate; $P F K-A S E=$ phosphofructokinase; $p y r=$ pyruvate; $s u c=$ succinate.

posed a valuable reduced dynamic model of glycolysis (denoted by $m T R M$ ), accounting for 9 species, 7 lumped reactions, and including 17 easily identifiable parameters. The rate constants of this model have been identified using the kinetic experimental data of Chassagnole et al., ${ }^{23}$ and Visser et al. ${ }^{6}$ The $m$ TRM model is presented in Table 3 . The model has been proved to adequately reproduce the cell glycolysis under oscillatory, or transient conditions according to the defined glucose concentration in the bioreactor, the total A(MDT)P cell energy resources, and the cell phenotype characteristics (concerning the ATPase enzyme activity, this essential enzyme being involved in the ATP utilization and recovery system). This is why the bioreactor (of 
model presented in Table 2) and the glycolysis dynamic models have to be considered together (Tables 2-3) when simulating the cell CCM.

\section{How glycolytic oscillations occur}

Oscillations in chemical systems represent periodic state variable (i.e. species concentrations) transitions in time.

According to Franck, ${ }^{24}$ spontaneous occurrence of self-sustained oscillations in chemical systems is due to the coupled actions of at least two simultaneous processes. Oscillations sourced in a so-called "oscillation node" (that is, a chemical species, or a reaction), on which concomitant rapid positive (perturbing) and slow negative (recovering) regulatory loops act. Because the coupling action between the simultaneous processes is mutual, the total coupling effect actually forms closed feedback loops for each kinetic variable involved. There exists a well-established set of essential thermodynamic and kinetic prerequisites for the occurrence of spontaneous oscillations. In short, according to Franck, ${ }^{24}$ these are the following:

1) Sustained oscillations can only occur in thermodynamically open systems far from equilibrium;

2) Oscillatory systems always consist of more than one degree of kinetic freedom, i.e. the description of their temporal behaviour requires a corresponding set of simultaneous differential equations;

3) Oscillations occur as a result of simultaneous feedback effects;

4) There exist extremely nonlinear relationships between the involved driving forces and driving fluxes or reactions; states;

5) Oscillatory systems always contain unstable

6) Oscillations are the result of mutual kinetic coupling between processes being otherwise independent from each other;

7) Once an oscillation occurs, it propagates in the whole reaction pathway;

8) Feedback occurs when a process acts kinetically upon itself; it therefore consists basically of a closed chain of action which causes the well-known effects of self-enhancement in the case of "positive feedback" (denoted by $\square$ ), and self-inhibition in the case of "negative feedback" (denoted by $\square$ ) respectively, in a non-systemic, or systemic feedback (i.e. rate constants depend on their own reaction products or reactants);

9) In general, there are four possible modes of coupling control loops leading to oscillatory processes, the positive $(\square)$ or the negative $(\square)$ feedbacks simultaneously acting on the synthesis and

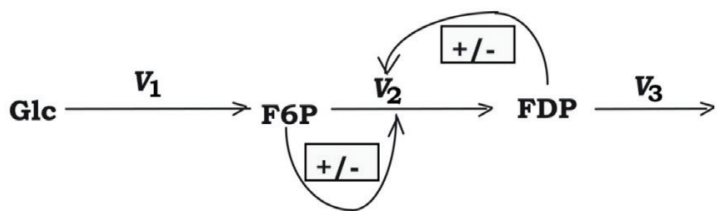

Fig. 2 - Chemical node inducing glycolytic oscillations (after ${ }^{27,28}$ ). Notations $\square$, and $\square$ denote the feedback positive or negative regulatory loops, respectively. Glc = glucose; $F 6 P=$ fructose-6-phosphate; $F D P=$ fructose-1,6-biphosphate; $V_{1}-V_{3}=$ reaction rates in Fig. 1 .

consumption of the oscillating "node species", that is: i) positive, and negative feedbacks; ii) positive feedback, and positive feedforward; iii) negative feedback and negative feedforward; iv) negative feedforward and positive feedforward. Such an oscillation "engine" of type (iv) is displayed in Fig. 2 for the glycolysis case;

10) Chemical oscillations exhibit positive and negative feedback simultaneously; according to the "principle of antagonistic feedback of chemical oscillators". Oscillations are understood as a consequence of an antagonistic interaction of a relatively fast acting positive feedback of labilizing tendency and a slower acting negative feedback of a recovering tendency for stabilizing the system;

11) Oscillations occurrence and characteristics depend not only upon the presence of both kinds of feedbacks but also upon the correct ratios of the time parameters (rate-constants) of the feedback loops involved.

In the glycolysis system case, extensive experiments have revealed that self-sustained oscillations are reported in a broad range of cell types ${ }^{1}$ (see for instance in Fig. 3 the plotted experimental glycolytic oscillations measured by Schaefer et al. ${ }^{25}$ in $E$. coli, and plotted by Chiarugi et al. ${ }^{26}$ ). As revealed by Termonia and Ross, ${ }^{27,28}$ Vermeer, ${ }^{29}$ and Chiarugi et al. ${ }^{26}$ glycolytic oscillations occurrence is due to the antagonistic action of two processes on regulating the $V_{2}$ reaction rate that converts F6P into FDP (see reaction scheme in Fig. 2). Valuable contributions to explain and model, on an experimental basis, the glycolytic oscillations "engine" and their self-control, have been done on E. coli, ${ }^{27,28,30-33}$ or on yeast. ${ }^{34}$

Glycolytic oscillation occurrence and characteristics (period) are influenced by both external (environmental) and internal (genomic) factors, that is: ${ }^{35,36}$

I) From one side it is the glucose (Glc) import driving force through the phosphotransferase (PTS)-system (Fig. 1) regulated and triggered by the external concentration of glucose $c_{G L C}^{\mathrm{ext}}=[\mathrm{Glc}] \mathrm{ext}$ 


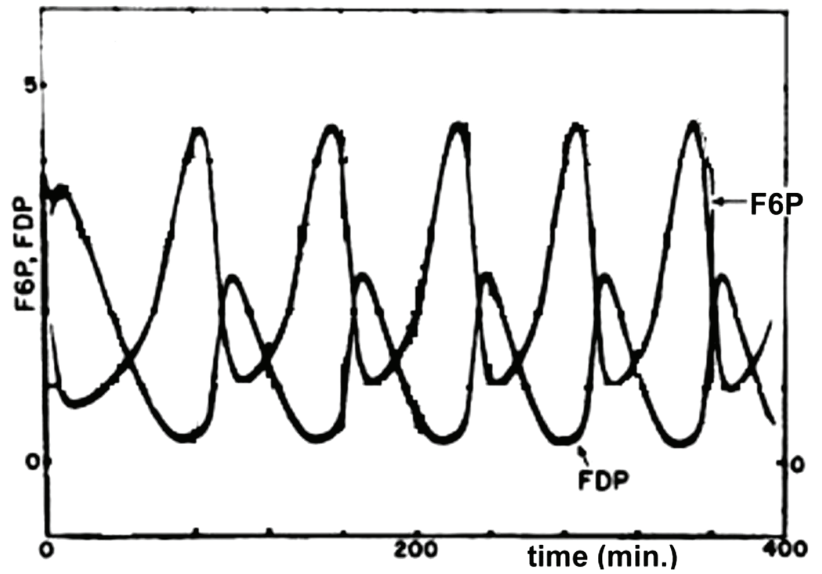

Fig. 3 - Experimentally measured glycolytic oscillations of fructose-6-phosphate (F6P) and fructose-1,6-bisphosphate (FDP). "In spite of white gaussian noise, the two plots have a clear constant period and amplitude, showing a stationary oscillatory pattern" in E. coli (adapted after Chiarugi et al. ${ }^{26}$ similar results have been obtained by Schaefer et al. ${ }^{25}$ ). Time axis in minutes. Concentrations are in $\mathrm{mM}$.

and by the PEP and PYR levels (see the $V_{1}$ rate expression in Table 3 ).

II) However, the Glc import and conversion to PYR requires important amounts of regenerable ATP, and a sufficiently rapid ATP to ADP conversion rate, as well as its quick regeneration (Fig. 1 and rate expressions in Table 3 ).

III) On the other hand, the limited A(MDT)P cell energy resources exist in the cell, slowing-down the Glc import if the ATP use/regeneration is not working "fast enough". ${ }^{36}$

IV) Additionally, due to the enzymes $A T P$-ase and AKase characteristics related to the bacteria genome and cell phenotype, a limited ATP conversion rate can sustain the glycolytic reactions, while the ATP recovery rate is limited by the enzymes participating in the A(MDT)P inter-conversion reactions ( $K$ and $k_{6}$, constants in Table 3 ).

V) At the same time, glycolysis being a systemic process with a complex regulatory structure, oscillations are also related to the rate constants of all the involved reactions. Similarly, Silva and Yunes $^{37}$ found that oscillations are only possible if the Glc concentration, and the maximum reaction rates controlled by the PFKase and GKase enzymes (involved in the $V_{1}$ reaction, i.e., the PTS import system) are within specific intervals.

VI) Consequently, among the glycolytic oscillation factors, it is natural to approach in this study in the first place the influence of the external factor [Glc]ext, and of some internal factors, such as the $k_{6}$ rate constant (belonging to the $\mathrm{A}(\mathrm{MDT}) \mathrm{P}$ inter-conversion system) on the glycolytic oscillations. The total [AMDTP] level was kept constant in this study to highlight the influence of the other mentioned factors.

Roughly, the same conclusions have been underlined by Silva and Yunes ${ }^{37}$ (even if in the $S$. cerevisiae case): "It appears that glycolytic oscillations are focused on the maintenance of energy levels in the cell (negative regulation of PFKase by ATP) and thus the ability to limit the conversion into energy in situations where it is not needed. Therefore, it would be more advantageous to store it or deviate the flux towards other cell cycle activities such as cell division." Consequently, mutant cells with modified enzymes activity (especially PFKase, PKase, ATPase, AKase, GKase) will lead to a noticeable modification in the cell metabolism.

Here it is important to mention the works ${ }^{30,38}$ dealing with explaining specific regulation of the glucose influx by PTS system in E. coli glycolytic, modelled in detail by Chassagnole et al. ${ }^{23}$ and Visser et al., ${ }^{6}$ and also the biochemical interactions among the PTS system, and the components of the ATP regeneration pathway (e.g. PFKase, PKase), used to evaluate the dynamic behavior of the glycolytic pathway of $E$. coli under steady-state conditions. Valuable contributions to model the glycolytic oscillations related to the system characteristics and environmental conditions have been reported. ${ }^{27,28,30-33}$ The advantage of the mTRM model of Maria ${ }^{4}$ is its capacity to reproduce glycolytic oscillations using a reduced kinetic model but still preserving the essential glycolytic and environmental parameters with a major influence on the process (see the next chapter on oscillation conditions).

\section{Simulation of some oscillation conditions}

By adopting the glycolysis kinetic model of Maria $^{4}$, one can determine, by repeated simulations, the cell external and internal conditions leading to glycolytic oscillation occurrence. In simulations, one considers the E. coli cell growing conditions of the semi-continuous bioreactor of Chassagnole et $a l{ }^{23}$ given in Table 1 (using sparging air in excess, and necessary nutrients for a cell culture equilibrated growth). The main mass balance equations of the bioreactor and glycolysis dynamic model are presented in Table 2. To obtain the model solution with enough precision, a low-order stiff integrator ("ODE23S" routine) of the Matlab ${ }^{\text {TM }}$ package was used.

Simulations were made for the cell culture conditions given in Table 1, and for cells with [AMDTP]total $=5.82 \mathrm{mM}^{4,23}$ Following the discussion in the previous chapter on oscillations occurence, the influence of two main factors is studied here, that is: 
i) [Glc]ext (related to the bioreactor operating conditions);

ii) $k_{6}$ reaction rate (determined by the ATP-ase characteristics, related to the cell phenotype);

iii) all other reaction rate constants, and [AMDTP] level were kept unchanged during simulations of values given in Tables 1, 3 .

Simulations were conducted in an exhaustive way, by covering the ranges of the initial [Glc]ext $=$ [0.01-1] $\mathrm{mM}$ (at $t=0$ ), and $k_{6}=[0.1-20] \mathrm{min}^{-1}$.

Table 1 -Operating conditions of the Chassagnole et al. ${ }^{23}$ semi-continuous bioreactor with suspended E. coli cell culture used to simulate the glycolytic oscillation occurrence

\begin{tabular}{ll}
\hline \multicolumn{1}{c|}{ Parameter } & \multicolumn{1}{c}{ Value } \\
\hline Biomass concentration $\left(C_{x}\right)$ & $\begin{array}{l}8.7 \mathrm{gDW} \mathrm{L}{ }^{-1} \text { culture volume } \\
\text { Cell content dilution rate }(D)\end{array}$ \\
$\begin{array}{l}1.667 \cdot 10^{-3} \mathrm{~min}^{-1} \\
\text { Culture dilution rate }\end{array}$ & $\begin{array}{l}1.667 \cdot 10^{-3} \mathrm{~min}^{-1} \text { (adjusted to } \\
\left(F_{L} / V_{L}\right)\end{array}$ \\
$\begin{array}{l}\text { Glucose feeding solution } \\
\text { concentration } c_{G L C}^{\text {feed }}\end{array}$ & $200 \mathrm{mM}$ (this paper). \\
Biomass density $\left(\rho_{x}\right)$ & $565.5 \mathrm{gDW}(\mathrm{L} \text { cytosol) })^{-1}$ \\
\hline
\end{tabular}

Measured [AMDTP]total

(assumed to be constant

$5.82 \mathrm{mM}$

in our simulations)
The results are presented in Table 4, and plotted in Fig. 4. These simulation results lead to several model-based conclusions:

I) Oscillations are basically determined by the external level of [Glc] (triggering the glucose import into the cell) but also, for a certain [AMDTP], total energy resources level in the cell (assumed to

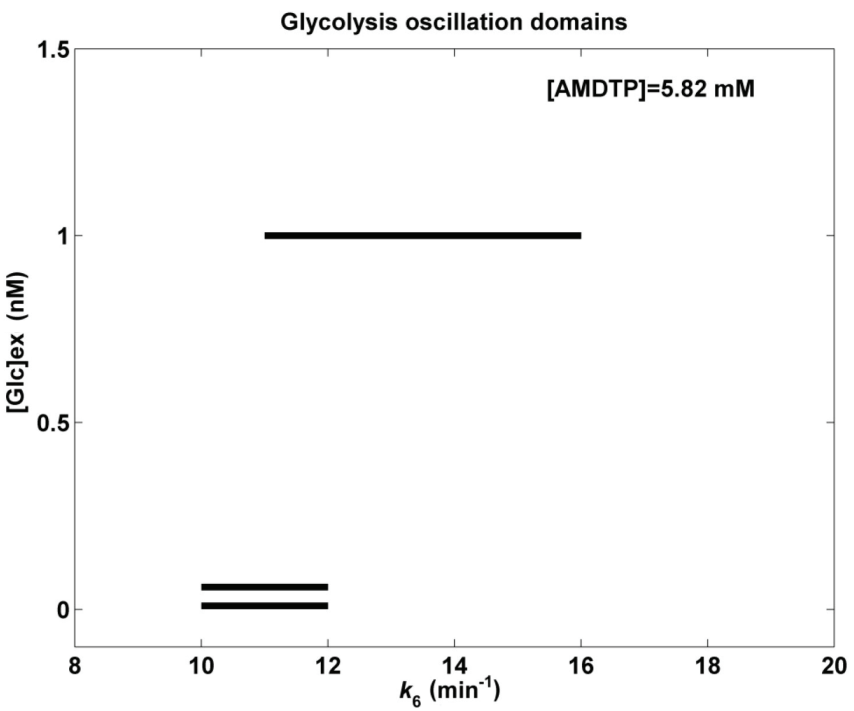

Fig. 4-Glycolytic stationary oscillation domains (thick lines) in E. coli in the plane [Glc]ext (at $t=0)$, and $k_{6}$ for the bioreactor operating conditions in Table 1 [AMDTP] $=5.82 \mathrm{mM}$

Table 2 -Bioreactor and glycolysis mass balance eqns. for the kinetic model of Maria

\begin{tabular}{|c|c|}
\hline Species mass balance & Auxiliary relationships \\
\hline$\frac{\mathrm{d} c_{G L C}^{e x t}}{\mathrm{~d} t}=D\left(c_{G L C}^{\text {feed }}-c_{G L C}^{e x t}\right)-\frac{C_{x}}{\rho_{x}} V_{1}$ & $\begin{array}{l}\text { i) Cell species initial concentrations are those measured by Chassagnole et al., }{ }^{23} \text { that is } \\
\text { (in } \mathrm{mM} \text { ): }\end{array}$ \\
\hline$\frac{\mathrm{d} c_{F 6 P}}{\mathrm{~d} t}=V_{1}-V_{2}-D c_{F 6 P}$ & $c_{G L C}^{e x t}(t=0)=$ tried reference value of $0.0557 \mathrm{mM}$, or $1 \mathrm{mM}$, \\
\hline$\frac{\mathrm{d} c_{F D P}}{\mathrm{~d} t}=V_{2}-V_{3}-D c_{F D P}$ & $c_{F G P}(t=0)=0.600325977$ \\
\hline$\frac{\mathrm{d} c_{P E P}}{\mathrm{~d} t}=2 V_{3}-V_{4}-D c_{P E P}$ & $c_{F D P}(t=0)=0.272961814$ \\
\hline$\frac{\mathrm{d} c_{P Y R}}{=}=V_{4}-V_{5}-D c_{P Y R}$ & $c_{P E P}(t=0)=2.67294507$ \\
\hline & $c_{P Y R}(t=0)=2.67061526$ \\
\hline & $c_{A T P}(t=0)=4.27$ (constant) \\
\hline & ii) $c_{A M P}+c_{A D P}+c_{A T P}=c_{A M D T P}=$ constant; ${ }^{27,28}$ \\
\hline $\mathrm{d} c_{A T P}$ & $\begin{array}{l}\text { iii) } c_{A D P} \text { results from solving the thermodynamic equilibrium relationship } \\
c_{A T P} c_{A M P}=K c_{A D P}^{2} \text {, that is: }\end{array}$ \\
\hline$-V_{6}-D c_{A T P}$ & $c_{A D P}^{2} \frac{K}{c_{A T P}}+c_{A D P}-c_{A M D T P}+c_{A T P}=0$ \\
\hline & iv) product formation from PYR has been neglected from this model; \\
\hline & v) biomass concentration $\left(C_{x}\right)$ is assumed to be quasi-constant. \\
\hline
\end{tabular}


Table 3 -Glycolysis kinetic model mTRM of Maria ${ }^{4}$ and its parameters (the units are in $\mathrm{mM}$, min)

Reaction $V_{1}$

$$
\begin{aligned}
& G L C+P E P \rightarrow F 6 P+P Y R \\
& P Y R+A T P \rightarrow P E P+A D P+H \\
& G L C+A T P \rightarrow F 6 P+A D P+H \\
& V_{1}=r_{P T S}=\frac{\rho_{x}}{C_{x}} \cdot \frac{r_{P T S}^{\max } c_{G L C}^{e x t} c_{P E P} / c_{P Y R}}{\left(K_{P T S, a 1}+K_{P T S, a 2} \frac{c_{P E P}}{c_{P Y R}}+K_{P T S, a 3} c_{G L C}^{e x t}+c_{G L C}^{e x t} \frac{c_{P E P}}{c_{P Y R}}\right)\left(1+\frac{c_{G 6 P}^{n_{P T S, G 6 P}}}{K_{P T S, G 6 P}}\right)}
\end{aligned}
$$

\section{Parameters}

$c_{G 6 P}=k c_{F 6 P} ;$

$r_{P T S}^{\max }=308.8587$

$K_{P T S, a 1}=1.0260$

$K_{P T S, a 2}=3740.091$

$K_{P T S, a 3}=5911.072$

$K_{P T S, G 6 P}=$ absent

$n_{P T S, G 6 P}=0$

$k=5.8$

\section{Parameters}

\section{Reaction $V_{2}$}

$\delta=1.0437$

$F 6 P+A T P \rightarrow F D P+A D P+H$

$V_{2 m}=0.062028$

$V_{2}=r_{P F K}=\frac{\left(V_{1} / V_{2 m}\right) c_{F G P}^{\delta}}{\left(K_{2 m}^{\delta}+K_{2 m}^{\delta}\left[\frac{K_{R}^{A M P}}{K_{T}^{A T P}}\right]^{n}\left(\frac{c_{A T P}}{c_{A M P}}\right)^{n}+c_{F G P}^{\delta}\right)}$

$K_{2 m}=6.16423$

$K_{R}^{A M P}=25 \mu \mathrm{M}$

$K_{T}^{A T P}=60 \mu \mathrm{M}$

\section{Reaction $V_{3}$}

$\mathrm{FDP}+2 \mathrm{ADP}(+2 \mathrm{NAD}+2 \mathrm{P}) \rightleftharpoons 2 \mathrm{PEP}+2 \mathrm{ATP}\left(+2 \mathrm{NADH}+2 \mathrm{H}+2 \mathrm{H}_{2} \mathrm{O}\right)$

$V_{3}=k_{3} c_{F D P}^{\alpha}-k_{3 p} c_{P E P}^{\beta}$

\section{Parameters}

$k_{3}=73.63477$

$k_{3 p}=337.0371$

$\alpha=0.05$

$\beta=3$

Reaction $V_{4}$

Parameters

$P E P+A D P+H \rightarrow P Y R+A T P$

$\gamma=1.33188$

$V_{4}=r_{P K}=\frac{\left(V_{1} / V_{4 m}\right) c_{P E P}^{\gamma}}{\left(K_{4 m}^{\gamma}+K_{4 m}^{\gamma}\left[\frac{K_{R}^{F D P}}{K_{T, P K}^{A T P}}\right]^{m}\left(\frac{c_{A T P}}{c_{F D P}}\right)^{m}+c_{P E P}^{\gamma}\right)}$

$m=4$

$V_{4 m}=0.13336$

$K_{4 m}=1.14644$

$K_{R}^{F D P}=0.2 \mathrm{mM}$

$K_{T, P K}^{A T P}=9.3 \mathrm{mM}$

Reaction $V_{5}$

$P Y R \rightarrow \operatorname{products}(A C C O A, C I T, S U C C, L A C, E T O H, A C, \ldots)$

\section{Parameters}

$V_{5}=\frac{k_{5} c_{P Y R}^{n_{\text {consum }, P Y R}}}{\mathrm{~K}_{\text {consum, PYR }}+c_{P Y R}}$

$k_{5}=693.3544$

$K_{\text {consum }, P Y R}=395.525$

$n_{\text {consum }, P Y R}=2.68139$

\section{Parameters}

$K=1$;

$k_{6}=4025.351$

$c_{A T P} c_{A M P}=K c_{A D P}^{2} ; A T P \rightarrow A D P+H ; V_{6}=k_{6} c_{A T P}$

Obs.: Termonia and Ross ${ }^{27,28}$ indicated experimental evidence of a very fast reversible reaction catalysed by AKase, the equilibrium being quickly reached.

Obs.: Other values of $k_{6}$ are also possible (to be investigated), according to the microorganism phenotype (characteristics of the gene encoding the enzyme ATPase that catalyse this reaction). 

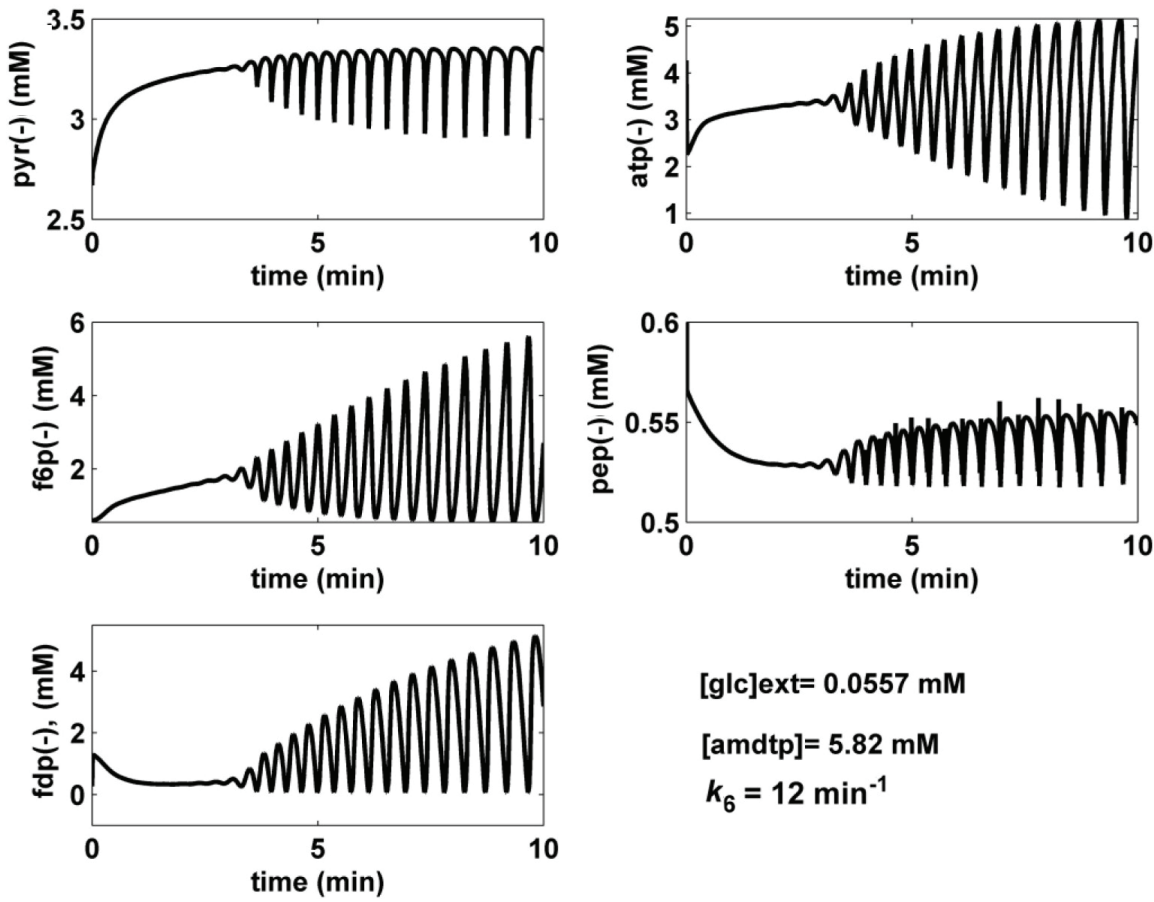

[glc]ext $=0.0557 \mathrm{mM}$

[amdtp] $=5.82 \mathrm{mM}$

$k_{6}=12 \mathrm{~min}^{-1}$
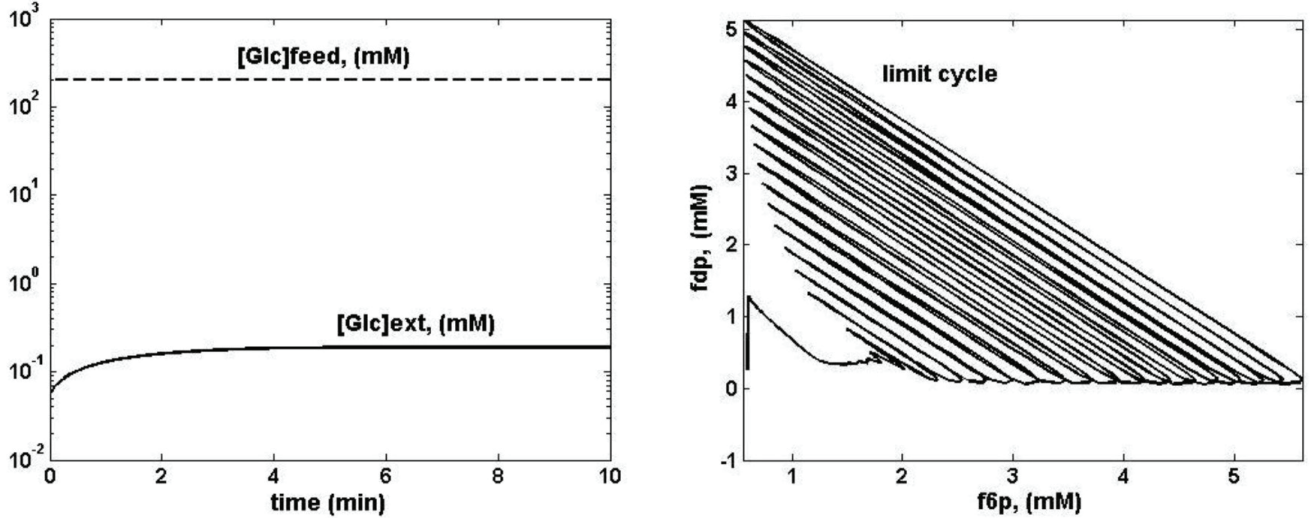

Fig. 5 - Simulated glycolytic stationary oscillations in E. coli and [Glc] dynamics in the bioreactor for the operating conditions in Table $1\left([A M D T P]=5.82 \mathrm{mmol} \mathrm{L} L^{-1}, D=1.667 \cdot 10^{-3} \mathrm{~min}^{-1}\right)$. Identified parameters inducing oscillations are: $[$ Glc]ext $=0.0557 \mathrm{mM}$ (at $t=0) ; k_{6}=12 \mathrm{~min}^{-1}$, approx. period of $0.33 \mathrm{~min}$. Notations: [Glc]ext = glucose concentration in the cell environment (bulk phase); [Glc]feed = glucose concentration in the feeding solution. (Bottom-right) The limit glycolytic cycle in [fdp,f6p] terms.

be quasi-constant in the present case study), are determined by the ATP to ADP conversion rate, and ATP regeneration rate (reflected here by $k_{6}$, and $K$ constants of Table 3 ).

II) Oscillations occur for low [Glc]ext but with a slow Glc import, due to relatively low $k_{6}$ constant values (i.e., a cell with a slow ATP conversion to ADP and ATP recovery).

III) By contrast, high levels of [Glc]ext, triggering high rate import, produce glycolytic oscillations for larger values of $k_{6}$, due to the limited ATP recovery rate ( $k_{6}$ being also related to the $K$ constant governing the AMDTP pathway). Eventually, for too small, or too large $k_{6}$ values, the glycolysis reaches its steady-state.

IV) The glycolytic oscillation domains in Fig. 4 , plotted in terms of $k_{6}$ and [Glc]ext, are very nar- row, revealing their high sensitivity with respect to the inducing factors, and their poor stability (as proved by the limit cycles plotted in Figs. 5-6). As expected, such a result indicates that oscillations stability is also dependent on the microorganism characteristics. For instance, by contrast, the glycolytic oscillations in yeast have been proved ${ }^{39,40}$ to be very robust even in the presence of environmental noise, "oscillations being a side-effect of the tradeoffs between robustness and regulatory efficiency of the feedback control of the autocatalytic reaction network".

V) As may be observed in Table 4, the increasing values of $k_{6}$ have, as a consequence, a slight decrease in the oscillation period until oscillation disappearance. This effect is better illustrated in Fig. 6 obtained for [Glc]ext $=1 \mathrm{mM}$ (at $t=0)$, and 

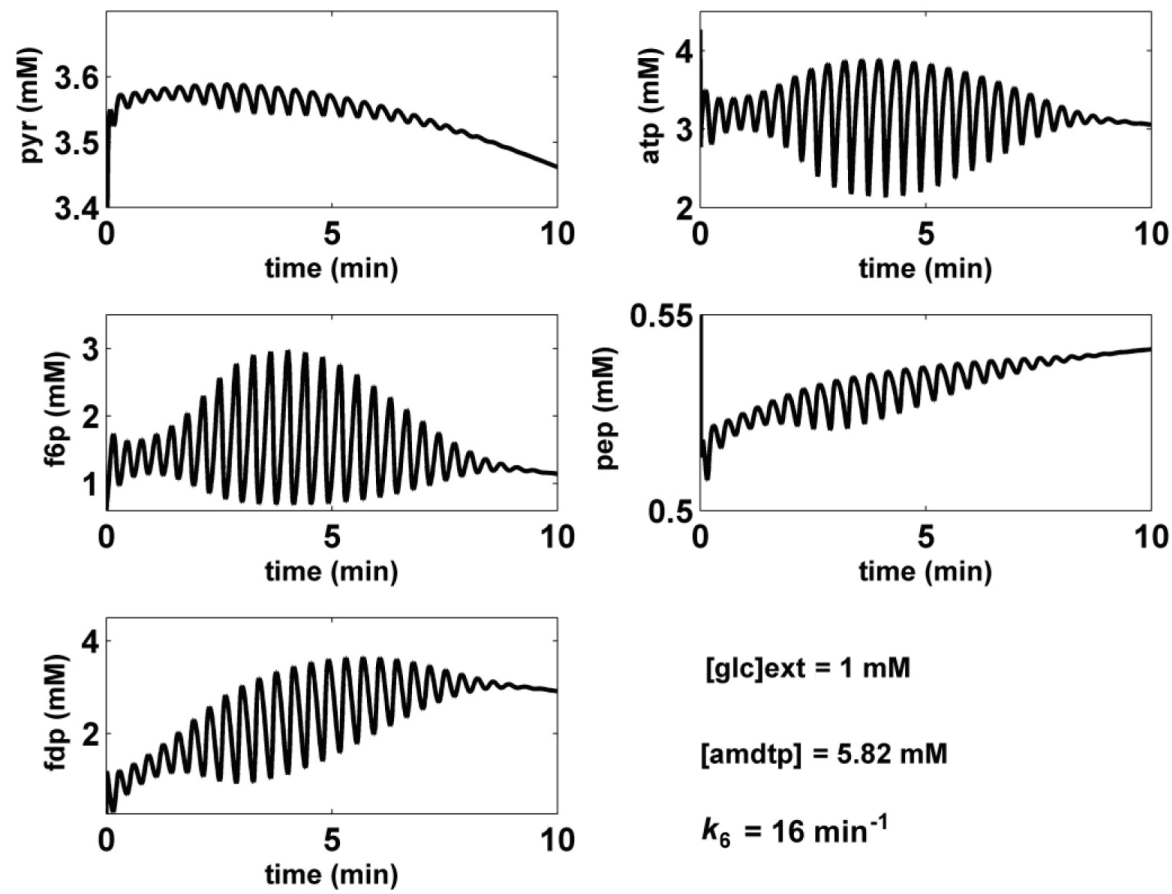

[glc]ext $=1 \mathrm{mM}$

[amdtp] $=5.82 \mathrm{mM}$

$k_{6}=16 \mathrm{~min}^{-1}$
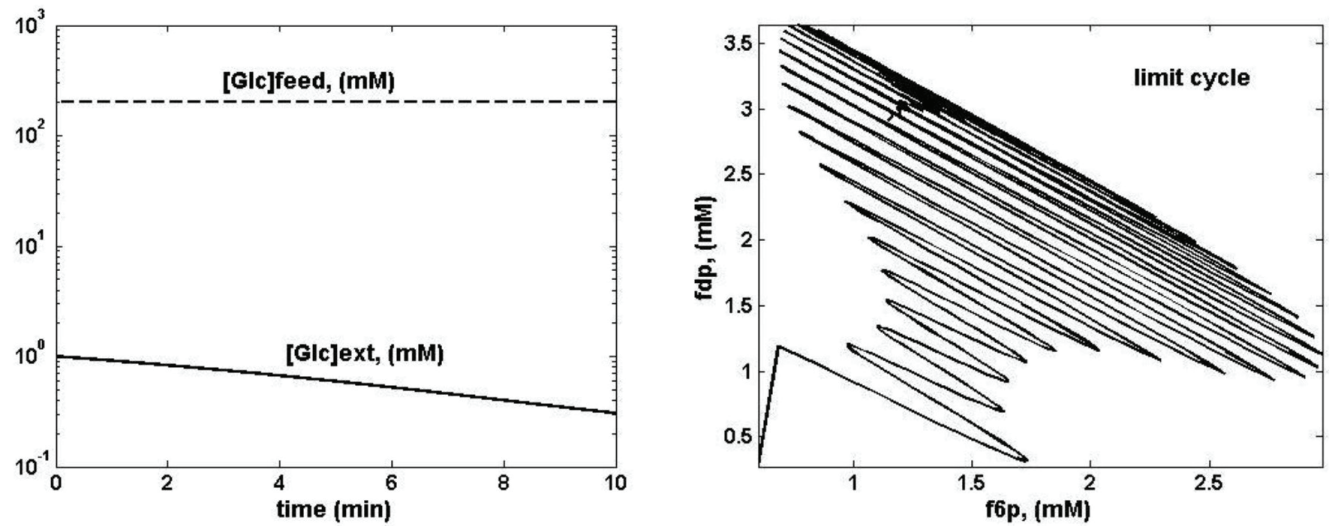

Fig. 6 - Simulated glycolytic stationary oscillations in E. coli and [Glc] dynamics in the bioreactor for the bioreactor operating conditions in Table $1\left([A M D T P]=5.82 \mathrm{mmol} \mathrm{L}{ }^{-1}, D=1.667 \cdot 10^{-3} \mathrm{~min}^{-1}\right)$. Identified parameters inducing oscillations are: [Glc]ext $=$ $1 \mathrm{mM}($ at $t=0) ; k_{6}=16 \mathrm{~min}^{-1}$, approx. period of $0.5 \mathrm{~min}$. Notations: [Glc]ext = glucose concentration in the cell environment (bulk phase); [Glc]feed = glucose concentration in the feeding solution. (Bottom-right) The limit glycolytic cycle in [fdp,f6p] terms.

$k_{6}=16 \mathrm{~min}^{-1}$, with an oscillation period of ca. 0.5 min. These oscillations are amortized and, eventually disappear (plots for larger operating times are not presented here) due to the sharp decline of [Glc]ext from the initial $1 \mathrm{mM}$ level (Fig. 6 down-left), due to its consumption by the cells, and its washout.

VI) For comparison, the simulation result plotted in Fig. 5, which was obtained for [Glc]ex = $0.0557 \mathrm{mM} ; k_{6}=12 \mathrm{~min}^{-1}$, presents a smaller oscillation period of ca. $0.33 \mathrm{~min}$, and a higher stability due to a smaller Glc environmental level and a higher ATP use/recovering rate. Table 4 also reveals that oscillation period takes values in the range of 0.4-0.9 min, being smaller as $k_{6}$ is bigger, and [Glc] ext is smaller. For comparison, experimentally determined glycolytic oscillations present periods of ca. $0.2 \mathrm{~min},{ }^{1}$ or $2-100 \mathrm{~s},{ }^{35} 15 \mathrm{~s},{ }^{37}$ or $1-20 \mathrm{~min},{ }^{36}$ up to $3 \mathrm{~h},{ }^{41}$ or 0.2 min to hours. ${ }^{30}$

Even if the checked time-interval, oscillation period, and bioreactor conditions of our simulations given in Fig. 5 are different of those experimentally checked in Fig. 3 (not mentioned by the authors), the theoretical curve shapes for FDP and F6P are similar to the experimental ones, even if the timescale of the abscissa is very different (400 $\mathrm{min}$ in Fig. 3, compared to $10 \mathrm{~min}$ in Fig. 5). The slight increase in the amplitude of oscillations of FDP and F6P are similar to the simulation results of Selkov, ${ }^{33}$ Bier et al.,${ }^{36}$ Elias, ${ }^{42}$ de la Fuente, ${ }^{43}$ etc.

VII) Also noteworthy are the dynamics of the [Glc]ext in the bioreactor bulk phase (a semi-continuous bioreactor with suspended $E$. coli cell culture) 
Table 4 - Some cell external and internal conditions leading to glycolytic oscillations occurrence in the E. coli cells. Total $[A M D T P]=5.82 \mathrm{mM}$ [nominal conditions of Chassagnole et al. ${ }^{23}$ and Maria ${ }^{4}$ presented in Table 1]. NO = evolution to quasi-steady-state with no oscillations.

\begin{tabular}{|c|c|c|}
\hline $\begin{array}{c}{[\mathrm{Glc}] \operatorname{ext}(\text { at } t=0),} \\
(\mathrm{mM})\end{array}$ & $k_{6}\left(\min ^{-1}\right)$ & $\begin{array}{c}\text { Approx. } \\
\text { oscillation } \\
\text { period (min.) }\end{array}$ \\
\hline \multirow{4}{*}{0.01} & $<1$, and $1-9$ & NO \\
\hline & 10 & 0.67 \\
\hline & 12 & 0.5 \\
\hline & $>13$ & $\mathrm{NO}$ \\
\hline \multirow{5}{*}{0.0557} & $<3$, and $3-7$ & NO \\
\hline & 10 & 0.66 (nominal) \\
\hline & 11 & 0.5 \\
\hline & 12 & 0.4 \\
\hline & $>13$ & NO \\
\hline \multirow{8}{*}{1} & $<11$ & NO \\
\hline & 11 & 0.9 \\
\hline & 12 & 0.8 \\
\hline & 13 & 0.7 \\
\hline & 14 & 0.6 \\
\hline & 15 & 0.5 \\
\hline & 16 & 0.5 (amortized) \\
\hline & $>17-19$ & $\mathrm{NO}$ \\
\hline
\end{tabular}

in the two operating cases. As expected, when the initial concentration of Glc is small $(0.0557 \mathrm{mM})$, the high Glc level in the feed $(200 \mathrm{mM})$ ensures a relatively quick fulfilment of the reactor steadystate conditions (of $0.188 \mathrm{mM}$ in Fig. 5 bottom-left). By contrast, when the initial concentration of Glc is higher $(1 \mathrm{mM})$, the reactor transition toward the steady-state (of $0.28 \mathrm{mM}$ in Fig. 6 bottom-left) is slower.

As for all in silico analyses, the results are strongly dependent on the used model quality.

To summarize, simulations varying the considered search variables in Table 4, and Fig. 4, clearly reveal the overwhelming importance of the environmental level of Glc, and of cell phenotype (that is, cell genomic and phenotype factors controlling the [AMDTP] total energy resources level, the glycolytic synthesis and regulation reactions, and especially the activity of enzymes involved in the A(MDT)P inter-conversion system). At the same time, glycolysis being a systemic process, with a complex regulatory structure, oscillations are also related to the rate constants of all the involved reactions and their appropriate ratios.
For the defined input data and model hypotheses, one can conclude that the derived results are conclusive enough. That is mainly because the used glycolysis $m T R M$ model presents very good adequacy vs. independent experimental data used for its identification by Maria. ${ }^{4}$ Consequently, one may conclude that the in-silico analysis results present satisfactory confidence and relevance. Of course, other variables, not accounted for in the model (cell characteristics reflected in the model constants) can influence the location of the problem solution. Subsequent experimental checks can validate the estimated glycolytic stationary oscillation domains in $E$. coli and, eventually, in the case of inconsistencies, they will lead to the model updating/completions for correcting its adequacy in order to perform future in silico analyses.

\section{Conclusions}

The use of reduced kinetic models describing the dynamics of complex metabolic pathways is a continuous challenging subject when developing structured cell simulators for various applications (flux analysis, target metabolite synthesis optimization, in silico re-programming of the cell metabolism for microorganism design purposes, bioreactor and bioprocess optimization). As exemplified by the E. coli glycolysis case study, the reduced $m T R M$ model, of simple and easily adaptable structure to various cell cultures, can be used in quick analyses of the cell metabolism, such as substrate utilization, oscillation occurrence, or structured interpretation of metabolic changes in modified cells.

Reduced structured glycolysis models of satisfactory adequacy for the key-species are preferred to other semi-empirical or very extended models, being easily included in modular cell simulation platforms used for solving various problems, such as: analysis of cell adaptation to certain environmental conditions; simulation of genetic regulatory circuits controlling the synthesis of some target metabolites; simulation of metabolic flux distribution and its dynamics under transient regimes; in silico reprogramming of some metabolic pathways to design new microorganisms. 5

Derivation of reduced kinetic structures to describe some parts of the cell core metabolism is worth the associated identification effort, due to the considerable reduction in the model parameterization (e.g., 17 parameters in the mTRM model vs. 127 in the Chassagnole et al..$^{23}$ model), while preserving a fair adequacy over a wide experimental domain. Besides, when cell characteristics change significantly, the reduced model can be upgraded quickly using the available experimental informa- 
tion. Thus, the cell metabolic process complexity appears to be described by a succession of "locally" reduced models "enfolded" on the real process long time-interval dynamics. ${ }^{4,11}$

Being quite versatile, the reduced $m T R M$ model includes enough information to reproduce not only the cell energy potential through the total A(MDT)P level, but also the role played by ATP/ ADP ratio as a glycolysis driving force. The model can also reproduce the oscillatory behaviour depending on the external/internal conditions, as well as situations when homeostatic conditions are not fulfilled.

The glycolysis core model can be easily extended by including any complex synthesis and regulatory pathway deriving from the main carbon uptake stream (e.g. CCM, succinate, SUCC, amino-acids production; Maria $\left.{ }^{5,16}\right)$, without necessarily complicating the 'core' model with too many species and parameters of less importance for the target metabolite production.

\section{Abbreviations and notations}

$\begin{array}{ll}\text { 1,3DPG, PGP } & - \text { 1,3-diphosphoglycerate } \\ \text { 2PG } & - \text { 2-phosphoglycerate } \\ \text { 3PG } & - \text { 3-phosphoglycerate } \\ \text { AC } & - \text { acetate } \\ \text { AK-ASE } & - \text { adenylate kinase } \\ \text { AMDTP } & - \text { adenosin-(mono)(di)(tri)phosphate } \\ \text { ATP } & - \text { adenosin-triphosphate } \\ \text { ATP-ASE } & - \text { ATP monophosphatase } \\ \text { CIT } & - \text { citrate } \\ \text { DHAP } & - \text { dihydroxyacetonephosphate } \\ \text { ETOH } & - \text { ethanol } \\ \text { F6P } & - \text { fructose-6-phosphate } \\ \text { FDP } & - \text { fructose-1,6-biphosphate } \\ \text { PEP } & - \text { phosphoenolpyruvate } \\ \text { PFK-ASE } & - \text { phosphofructokinase } \\ \text { PK-ASE } & - \text { pyruvate kinase } \\ \text { PPP } & - \text { pentose-phosphate pathway } \\ \text { PTS } & - \text { phosphotransferase, } \\ & \quad \text { or phosphoenolpyruvate: } \\ \text { PYR } & - \text { glucose phosphotransferase system } \\ \text { SUCC, SUC } & - \text { suruvate } \\ \text { TCA } & - \text { tricarboxylic acid cycle } \\ \text { TF } & - \text { transcription factors } \\ \text { [.] } & - \text { concentration } \\ \text { FOR } & - \text { formate } \\ \text { FUM } & - \text { fumarate } \\ \text { G3P, GAP } & - \text { glyceraldehyde-3-phosphate } \\ \text { G6P } & - \text { glucose-6-phosphate } \\ & - \text { a }\end{array}$

- glucose

GLCex, GLC [ext] - Glucose in the external environment

GLN - glutamine

HK-ASE - hexokinase

LAC - lactate

MAL - malate

mTRM - modified Termonia and Ross ${ }^{27,28}$ model

$\mathrm{NAD}(\mathrm{P}) \mathrm{H} \quad$ - nicotinamide adenine dinucleotide (phosphate) reduced

Pi - Phosphoric acid

$c_{j} \quad-$ species $j$ concentration

$C_{x} \quad-$ biomass concentration

D $\quad$ - cell content dilution rate (identical to the adjustable culture dilution rate, $\left.F_{L} / V_{L}\right)$

$F_{L} \quad-$ liquid feed flow rate in the bioreactor

$k_{j}, K_{j}, V_{2 m}, V_{4 m}, r_{j}^{\max }$ - rate and equilibrium constants

$t \quad$ - time

$V_{j} \quad-$ species $j$ reaction rates

$V_{L} \quad-$ liquid volume in the bioreactor

$\alpha, \beta, \gamma, \delta \quad-$ reaction orders

$\rho_{x}$

- biomass density

\section{References}

1. Madsen, M. F., Dano, S., Sorensen, P. G., On the mechanisms of glycolytic oscillations in yeast, FEBS Journal 272 (2005) 2648. doi: https://doi.org/10.1111/j.1742-4658.2005.04639.x

2. Wierschem, K., Bertram, R., Complex bursting in pancreatic islets: A potential glycolytic mechanism, J. Theor. Biol. 228 (2004) 513.

doi: https://doi.org/10.1016/j.jtbi.2004.02.022

3. Stephanopoulos, G., Simpson, T. W., Flux amplification in complex metabolic networks, Chem. Eng. Sci. 52 (1997) 2607.

doi: https://doi.org/10.1016/S0009-2509(97)00077-8

4. Maria, G., In silico derivation of a reduced kinetic model for stationary or oscillating glycolysis in Escherichia coli bacterium, Chem. Biochem. Eng. Quart. 28 (2014) 509. doi: https://doi.org/10.15255/CABEQ.2014.2002

5. Maria, G., Deterministic modelling approach of metabolic processes in living cells - a still powerful tool for representing the metabolic process dynamics, Newbury Park, California 91320 (USA), Juniper publ., 2017a.

6. Visser, D., Schmid, J. W., Mauch, K., Reuss, M., Heijnen, J. $J$., Optimal re-design of primary metabolism in Escherichia coli using linlog kinetics, Metab. Eng. 6 (2004) 378. doi: https://doi.org/10.1016/j.ymben.2004.07.001

7. Maria, G., A review of some novel concepts applied to modular modelling of genetic regulatory circuits, Newbury Park, California 91320 (USA), Juniper publ., 2017b.

8. Styczynski, M. P., Stephanopoulos, G., Overview of computational methods for the inference of gene regulatory networks, Comput. Chem. Eng. 29 (2005) 519.

doi: https://doi.org/10.1016/j.compchemeng.2004.08.029 
9. Hempel, D. C., Development of biotechnological processes by integrating genetic and engineering methods, Eng. Life Sci. 6 (2006) 443. doi: https://doi.org/10.1002/elsc.200620149

10. Heinemann, M., Panke, S., Synthetic Biology - putting engineering into biology, Bioinformatics 22 (2006) 2790. doi: https://doi.org/10.1093/bioinformatics/btl469

11. Maria, G., Luta, I., Structured cell simulator coupled with a fluidized bed bioreactor model to predict the adaptive mercury uptake by $E$. coli cells, Comput. Chem. Eng. 58 (2013) 98. doi: https://doi.org/10.1016/j.compchemeng.2013.06.004

12. Maria, G., Application of (bio) chemical engineering principles and lumping analysis in modelling the living systems, Curr. Trends Biomed. Eng. \& Biosc. 1, (2017b), ms. No. CTBEB.MS.ID.555566, https://juniperpublishers.com/ ctbeb/volume1-issue4-ctbeb.php

13. Lubert, S., Glycolysis, In: Biochemistry. (Fourth ed.), New York, Freeman W.H. Co., 1995, pp. 483-508.

14. Mathews, C. K., van Holde, K. E., Ahem, K. G., Biochemistry, New Jersey, Prentice Hall, 1999.

15. Calhoun, K. A., Swartz, J. R., Total amino acid stabilization during cell-free protein synthesis reactions, J. Biotechnol. 123 (2006) 193 doi: https://doi.org/10.1016/j.jbiotec.2005.11.011

16. Maria, G., Gijiu, C. L., Maria, C., Tociu, C., Interference of the oscillating glycolysis with the oscillating tryptophan synthesis in the E. coli cells, Comput. Chem. Eng. 108 (2018) 395. doi: https://doi.org/10.1016/j.compchemeng.2017.10.003

17. Reeves, R. E., Sols, A., Regulation of Escherichia coli phosphofructokinase in situ, Biochem. Biophys. Res. Commun. 50 (1973) 459. doi: https://doi.org/10.1016/0006-291X(73)90862-0

18. Bennet, B. D., Kimball, E. H., Gao, M., Osterhout, R., Van Dien, S. J., Rabinowitz, J. D., Absolute metabolite concentrations and implied enzyme active site occupancy in Escherichia coli, Nat. Chem. Biol. 5 (2009) 593. doi: https://doi.org/10.1038\%2Fnchembio.186

19. Flamholz, A., Noor, E., Bar-Even, A., Liebermeister, W., Milo, R., Glycolytic strategy as a tradeoff between energy yield and protein cost, Proc. Natl. Acad. Sci. U.S.A. 110 (2013) 10039 .

doi: https://doi.org/10.1073/pnas.1215283110

20. Alberton, K. P. E., Alberton, A. E., Di Maggio, J. A., Estrada, V. G., Diaz, M. S., Secchi, A. R., Simultaneous parameters identifiability and estimation of an $E$. coli metabolic network model, BioMed Res. Int., article ID= 454765 (2015). doi: https://doi.org/10.1155/2015/454765

21. Maria, G., A review of algorithms and trends in kinetic model identification for chemical and biochemical systems, Chem. Biochem. Eng. Quart. 18 (2004) 195.

22. Maria, G., Relations between apparent and intrinsic kinetics of programmable drug release in human plasma, Chem. Eng. Sci. 60 (2005) 1709. doi: https://doi.org/10.1016/j.ces.2004.11.009

23. Chassagnole, C., Noisommit-Rizzi, N., Schmid, J. W., Mauch, K., Reuss, M., Dynamic modeling of the central carbon metabolism of Escherichia coli, Biotechnol. Bioeng. 79 (2002) 53. doi: https://doi.org/10.1002/bit.10288

24. Franck, U. F., Feedback kinetics in physicochemical oscillators, Ber. Bunsenges. Phys. Chem. 84 (1980) 334. doi: https://doi.org/10.1002/bbpc.19800840407

25. Schaefer, U., Boos, W., Takors, R., Weuster-Botz, D., Automated sampling device for monitoring intracellular metabolite dynamics, Anal. Biochem. 270 (1999) 88. doi: https://doi.org/10.1006/abio.1999.4048
26. Chiarugi, D., Chinellato, M., Degano, P., Brutto, G. L., Marangoni, R., Feedbacks and oscillations in the virtual cell VICE, International Conference Computational Methods in Systems Biology (CMSB), Trento, Italy, Oct. 18-19, 2006, Proceedings (pp.93-107).

doi: https://doi.org/10.1007/11885191_7

27. Termonia, Y., Ross, J., Oscillations and control features in glycolysis: Numerical analysis of a comprehensive model, Proc. Natl. Acad. Sci. U.S.A. 78 (1981a) 2952. doi: https://doi.org/10.1073/pnas.78.5.2952

28. Termonia, Y., Ross, J., Oscillations and control features in glycolysis: Analysis of resonance effects, Proc. Natl. Acad. Sci. U.S.A. 78 (1981b) 3563. doi: https://doi.org/10.1073/pnas.78.6.3563

29. Vermeer, $S$., Spatio-temporal dynamics of glycolysis in an open spatial reactor, PhD Thesis, TU Magdeburg, 2008.

30. Diaz Ricci, J. C., ADP modulates the dynamic behavior of the glycolytic pathway of Escherichia coli, Biochem. Biophys. Res. Commun. 271 (2000) 244. doi: https://doi.org/10.1006/bbrc.2000.2603

31. Lavrova, A. I., Schimansky-Geiger, L., Postnikov, E. B., Phase reversal in the Selkov model with inhomogeneous influx, Phys. Rev. E79 (2009), paper 057102. doi: https://doi.org/10.1103/PhysRev

32. Verveyko, D. V., Verisokin, A. Y., Postnikov, E. B., Mathematical model of chaotic oscillations and oscillatory entrainment in glycolysis originated from periodic substrate supply, Chaos 27 (2017) paper 083104. doi:https://doi.org/10.1063/1.4996554

33. Selkov, E., Self-oscillations in glycolysis, Eur. J. Biochem, 4 (1968) 79. doi: https://doi.org/10.1111/j.1432-1033.1968.tb00175.x

34. Hald, B. O., Smrcinova, M., Sørensen, P. G., Influence of cyanide on diauxic oscillations in yeast, FEBS Journal 279 (2012) 4410 doi: https://doi.org/10.1111/febs.12030

35. Westermark, P. O., Lansner, A., A model of phosphofructokinase and glycolytic oscillations in the pancreatic $\beta$-cell, Biophys. J. 85 (2003) 126. doi: https://doi.org/10.1016/S0006-3495(03)74460-9

36. Bier, M., Teusink, B., Kholodenko, B. N., Westerhoff, H. V., Control analysis of glycolytic oscillations, Biophys. Chem. 62 (1996) 15. doi: https://doi.org/10.1016/S0301-4622(96)02195-3

37. Silva, A. S., Yunes, J. A., Conservation of glycolytic oscillations in Saccharomyces cerevisiae, Genet. Mol. Res. 3-5 (2006) 525.

38. Demin, O. V., Lebedeva, G. V., Kolupaev, A. G., Zobova, E. A., Plyusnina, T. Y., Lavrova, A. I., Dubinsky, A., Goryacheva, E. A., Tobin, F., Goryanin, I. I., Kinetic Modelling as a Modern Technology to Explore and Modify Living Cells, In: Ciobanu, G., Rozenberg, G., (eds.), Natural computing series, Springer, Berlin, 2004, pp. 59. doi: https://doi.org/10.1007/978-3-642-18734-6_4

39. Gehrmann, E., Glässer, C., Jin, Y., Sendhoff, B., Drossel, B., Hamacher, $K$., Robustness of glycolysis in yeast to internal and external noise, Phys. Rev. E84 (2011) 021913. doi: https://doi.org/10.1103/PhysRevE.84.021913

40. Chandra, F. A., Buzi, G., Doyle, J. C., Glycolytic oscillations and limits on robust efficiency, Science 333 (2011) 187 doi: https://doi.org/10.1126/science.1200705

41. Rapp, P., An atlas of cellular oscillators, J. Exp. Biol. 81 (1979) 281.

42. Elias, A. C., Escherichia coli: Dynamic analysis of the glycolytic pathway, $\mathrm{PhD}$ Thesis, Facultad de Bioquímica y Farmacia, Universidad Nacional de Tucumán. Argentina, 2010.

43. de la Fuente, I. M., Quantitative analysis of cellular metabolic dissipative, self-organized structures, Int. J. Mol. Sci. 11 (2010) 3540 doi: https://doi.org/10.3390/ijms11093540 